\title{
Arrhythmia Detection Based on Patient-Specific Normal ECGs Using Deep Learning
}

\author{
Shota Hori ${ }^{1}$, Toru Shono ${ }^{1}$, Keiji Gyohten ${ }^{1}$, Hidehiro Ohki ${ }^{1}$, Toshiya Takami ${ }^{1}$, Noboru Sato $^{2}$ \\ ${ }^{1}$ Oita University, Oita, Japan \\ ${ }^{2}$ Keiwakai Healthcare Group, Oita, Japan
}

\begin{abstract}
Most traditional studies regarding arrhythmia detection using electrocardiogram (ECG) have proposed general methods applicable to various patients. Because patients have their own unique ECG patterns, abnormalities undetected by general methods can be detected if a new arrhythmia detection method tailored to each patient is developed. Furthermore, the new method can effectively support doctors in their diagnosis if it can provide the basis for determining abnormalities. Herein, we propose an individualized ECG abnormality judgment method using an autoencoder and a convolutional neural network. This method allows the autoencoder to learn only normal waveforms that can be easily collected and obtains the characteristics of the individual's unique normal waveforms. Our method compares the features acquired from the ECG pattern to be analyzed with those of the normal waveform and determines whether they are normal or abnormal. In addition, we aim to construct a system that can demonstrate the basis for the judgment of whether a feature is normal or abnormal by showing the acquired features.
\end{abstract}

\section{Introduction}

In recent years, the need for automatic electrocardiogram (ECG) analysis to support physicians has increased. One of the approaches to achieve automatic ECG analysis is to use deep learning. This method is mainly based on supervised learning. Because this conventional method using supervised learning typically manages the data of multiple people for automatic analysis, its performance has become generic for many patients. ECG patterns vary by individual; therefore, an automatic analysis of ECGs tailored to individuals is expected. In addition, it is difficult to present the basis for judgments in automatic analysis. Conventional automatic ECG analysis is used to determine abnormal waveforms in short-time data, such as " 1 second" or " 1 beat" [1]. However, it can be difficult to analyze ECG patterns without observing their changes over time; as such, observations over a long time period are necessitated. Kiranyaz et al. proposed a patient-specific ECG classification based on supervised learning using a one-dimensional convolutional neural network (CNN) [2]. By learning a patient's individual ECG pattern and the ECG pattern common to each patient, the system performs an automatic ECG analysis that is specific to the individual. However, this system requires labeled ECG data, including those of arrhythmias, to be prepared for each individual. Because it may be difficult to collect large numbers of abnormal ECG patterns from individuals, the method is impractical to all patients. Elzen et al. proposed a method of representing dynamic networks as trajectories in a feature space to understand the temporal variation of dynamic networks [3]. In this method, the network at each moment is reduced to a low dimension using principal component analysis (PCA) and other methods, and the low-dimensional quantity is plotted continuously over time to represent the time change. In this study, we combined this method with feature extraction using an autoencoder to represent long-term changes in ECG patterns as trajectories. Using this method, we aim to construct an automatic ECG analysis system that can consider abnormalities over time using an autoencoder and a CNN to learn only normal waveforms that can be collected easily.

\section{Method and material}

This chapter describes a system that uses Autoencoder and CNN to determine whether an ECG is arrhythmic or not.

\subsection{Acquisition of ECG features}

In our method, ECG patterns are learned using an autoencoder and a CNN is used to acquire the essential features of the ECG pattern. The essential features are vectors represented in far fewer dimensions than the dimensions of the ECG pattern and can accurately reconstruct the original ECG pattern. The autoencoder was used because of three reasons. The first reason is to render the ECG pattern robust to noise. It is important to remove noise as it can result in misdiagnoses. The second reason is 
to visualize ECG features. By comparing the features of normal and abnormal waveforms in graphical form, we can obtain a basis for diagnosing the ECG pattern. Third, the autoencoder was trained with unsupervised learning. Without preparing abnormal ECG patterns, the system can be used by learning only normal waveforms that can be collected easily.

\subsubsection{Input and output data}

We used N-dimensional ECG waveform data as input and output. In the proposed method, we created input data by cropping out parts of the ECG pattern individually in an $\mathrm{N}$-dimensional width with the sliding window, as shown in Figure 1.

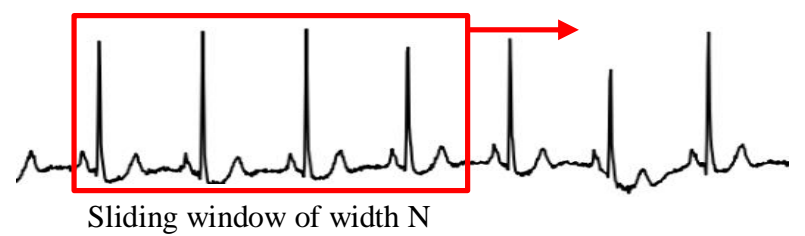

Figure 1. Input data creation method.

Using this method, the variability of the ECG pattern over a certain long-term period can be determined. Only ECG patterns diagnosed as normal in advance were used as input data for learning. The learning data were categorized into training and validation data. To evaluate the proposed method, not only normal waveforms, but also abnormal waveforms were included in the test data.

\subsubsection{Network structure}

We used an autoencoder to reduce the dimensionality of the $\mathrm{N}$-dimensional ECG waveform to T-dimensions and acquired the features. The network structure comprised six convolution layers, six deconvolution layers, three pooling layers, and three unpooling layers, as shown in Figure 2.

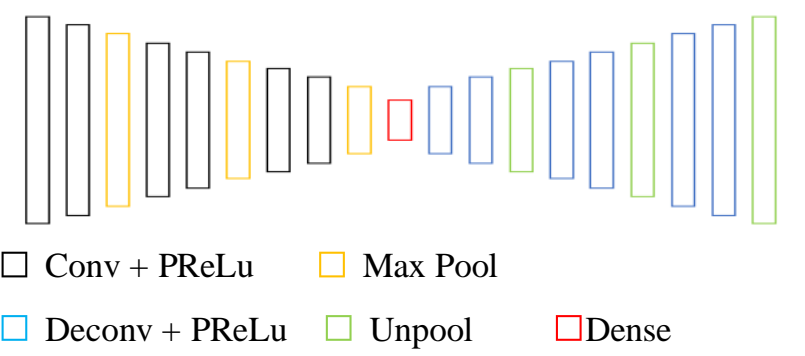

Figure 2. Network structure.

\subsection{ECG analysis}

After training the model with the training data, the features of all the learning data were acquired from the middle layer. Our method compares the features acquired from the training data with those acquired from the validation and test data using the nearest neighbor method and determines a threshold value. Subsequently, it assesses whether the input ECG pattern is arrhythmic.

\subsubsection{Nearest neighbor method}

Our method maps the T-dimensional features acquired from the middle layer onto the feature space and compares the training data with the validation and test data. Figure 3 shows the T-dimensional feature space. The Euclidean distances of the validation and test data to the closest training data were obtained and used as the measure of abnormality degree in the ECG pattern. Figure 4 shows the distances of all the validation and test data.

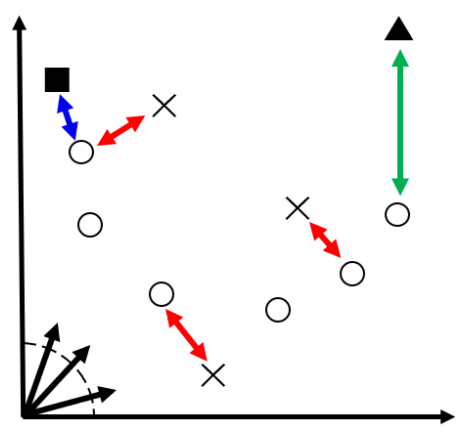

Figure 3. $\bigcirc$ : Training data. $\times$ : Validation data. $\square$ : Test data consisting of normal waveforms. $\boldsymbol{\Delta}$ : Test data comprising abnormal waveforms. Red, blue, and green lines indicate distances between respective data.

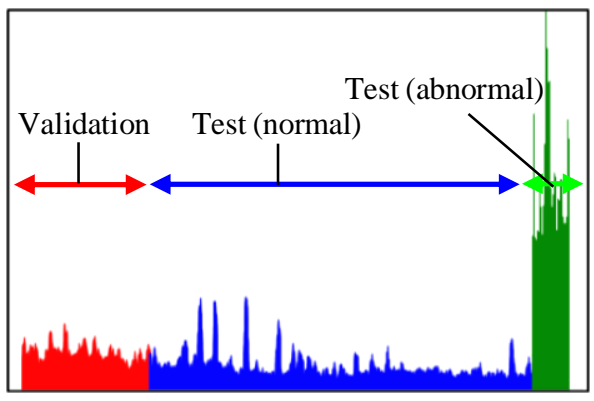

Figure 4. Distance to all validation and test data

\subsubsection{Threshold}

We determined whether the N-dimensional ECG data are arrhythmic by verifying the distances of the test data acquired in Section 2.2.1. This set was obtained from the window sliding $\mathrm{S}$ times to the right (as shown in Figure 1) and is represented by S-dimensional data, where each element represents the abnormality degree described in 
Section 2.2.1. We performed the following two steps:

1: If the distance is more than threshold $\mathrm{A}$, then the data are regarded as potentially abnormal.

2: Equation 1 shows the probability that the Sdimensional data are arrhythmic.

((Data judged to be abnormal by threshold A)/S).

If this probability is greater than or equal to threshold

$\mathrm{B}$, then the S-dimensional data are regarded a waveform reflecting arrhythmia.

These thresholds were calculated using the validation data.

We used the top $10 \%$ of distances in all validation data as threshold A and the top $0.5 \%$ of the probability determined in Step 2 for all validation data as threshold B. We verified for arrhythmia by applying this threshold to the test data.

\subsection{Visualizing Features}

We used PCA to dimensionally reduce the Tdimensional features acquired using the proposed method to two-dimensional features. We mapped two-dimensional features onto a two-dimensional graph and compared the trajectory of a normal waveform with that of an abnormal waveform. Figure 5 shows an example of the mapping result. This allows us to visually identify abnormal areas.

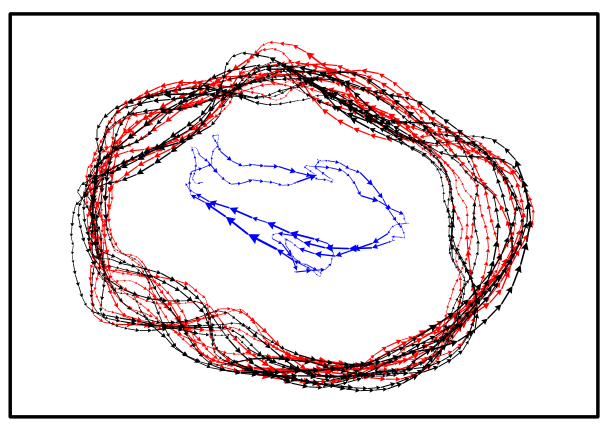

Figure 5. Feature paths of normal ECG for training (red), normal ECG for testing (black), and abnormal ECG for testing (blue).

\section{Experimental results}

This section describes the dataset to be used as ECG data and the method to evaluate the accuracy of the proposed method; additionally, the experimental results and discussions are provided.

\subsection{Dataset}

We used 30 ECG patterns from the MIT-BIH arrhythmia database [4]. This dataset contains 30-min ECG patterns of 48 individuals recorded using a Holter ECG recorder. Arrhythmias are classified as any heartbeat not in the S, V, F, or Q class, supraventricular ectopic beat, ventricular ectopic beat, fusion beat, and unknown beat based on the criteria recommended by the Association for the Advancement of Medical Instrumentation. These classes are attached to every $\mathrm{R}$-wave. The sampling frequency was $360 \mathrm{~Hz}$. Using the method described in Section 2.1.1, we acquired 30000 learning data from the beginning of each patient's ECG pattern. We set N, the dimension of the input, to 500 such that the input contains multiple R waves. Portions that include arrhythmias were excluded from the learning data; there were 29000 training data points and 1000 validation data. The test data comprised 3,000 normal and abnormal waveforms. Because the amount of abnormalities differed by individual, the amount of test data differed by individual as well. We prepared $\mathrm{S}$ data for each label of anomaly using the method described in Section 2.1.1. This was prepared for the number of anomalies.

\subsection{Evaluation method}

The results of the judgments obtained in the experiment were evaluated in terms of accuracy, precision, specificity, and F-values, which are typically used as accuracy indicators for binary classification. These indicators were obtained using Equations 2-6 with reference to Table 1.

Table 1. Four numerical values determined by binary classification.

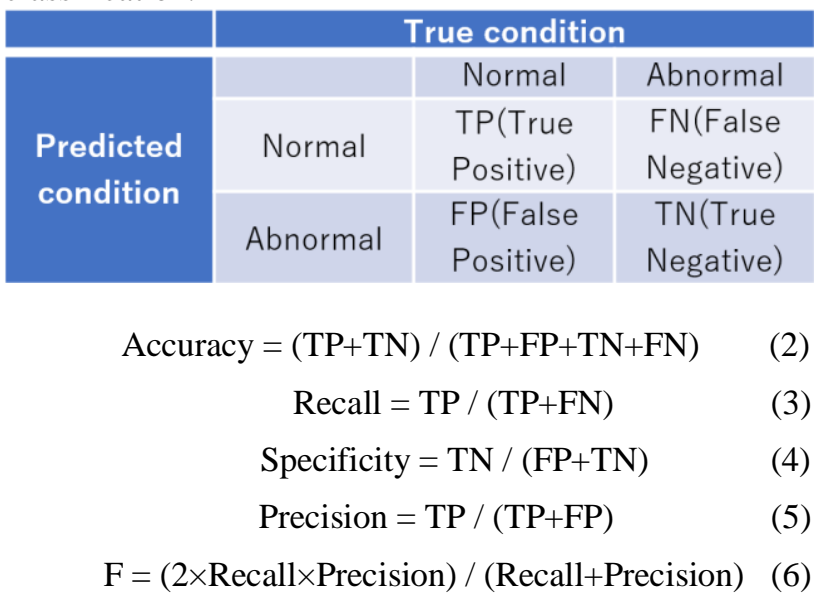

\subsection{Experimental results}

In our experiment, we defined the $\mathrm{T}$ and $\mathrm{S}$ dimensions as 20 and 150, respectively. We calculated the accuracy, precision, specificity, and F-values in a dataset of 30 individuals, and the results of the average of the 30 individuals are shown in Table 2. 
Table 2. Results for accuracy, precision, specificity and Fvalue.

\begin{tabular}{|c|c|c|c|}
\hline Accuracy & Precision & Specificity & F-measure \\
\hline 88.4 & 76.7 & 95.2 & 76.7 \\
\hline
\end{tabular}

The precision results show that the normal waveform was not accurately detected. This was because the normal waveform was not accurately restored owing to misalignment or noise, which deformed the shape of the normal waveform. Meanwhile, we identified the possibility of detecting abnormal waveforms from the results of specificity.

The feature visualization shows three patterns, as shown in Figures $6-8$.

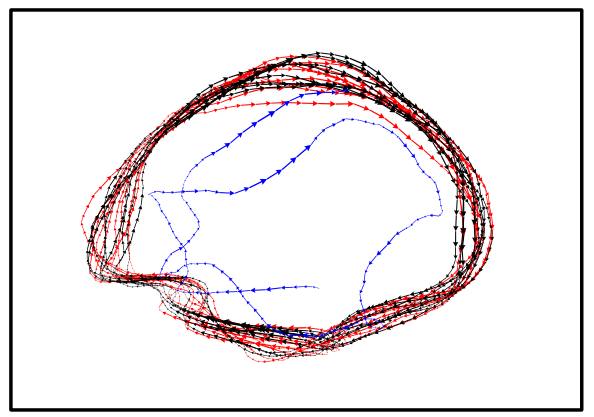

Figure 6. Pattern 1: Normal and abnormal trajectories are different.

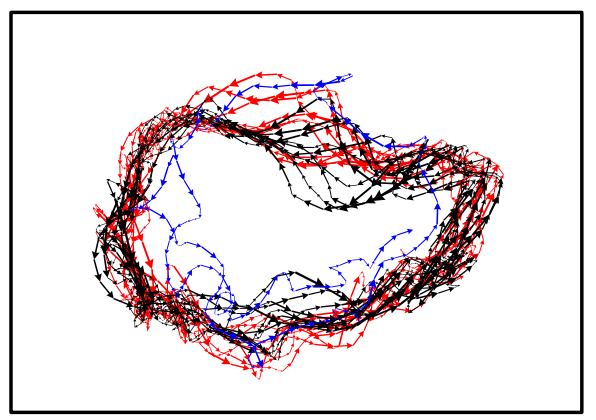

Figure 7. Pattern 2: Three vectors have the same trajectory.

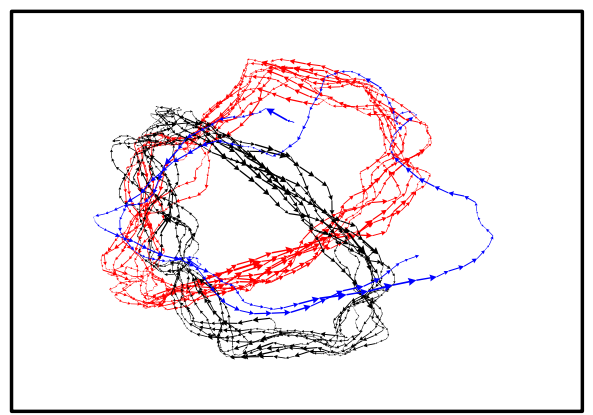

Figure 8. Pattern 3: Three vectors have different trajectories.

The result of pattern 1 shows the difference between normal and abnormal waveforms, which may provide a basis for judgment. Meanwhile, cases occurred where the visualization failed, as shown in patterns 2 and 3 . In addition to judgment inaccuracy, we considered that the visualization failed because the normal waveform was not accurately restored because of misalignment or noise, which deformed the shape of the normal waveform.

\section{Conclusion}

Herein, we focused on three conventional issues of automatic ECG analysis: (i) the conventional judgment of automatic classification is generic, (ii) the process of the analysis is a black box, and (iii) the arrhythmia caused by changes over time must be considered. We proposed an automatic ECG analysis system that can consider abnormality over time using an autoencoder and a CNN to learn only normal waveforms that can be easily collected. The experimental results demonstrated that the accuracy can be further improved and to the basis of the judgment can be analyzed by solving some problems. In addition, we successfully detected arrhythmias in people wearing pacemakers, otherwise undetectable using conventional generic determination methods. Two issues must be addressed in the future. The first is to reduce the baseline shift and noise. We attempted to solve this problem by introducing white noise. The second is to analyze a method for determining the threshold value. We plan to use outlier and anomaly detection methods to determine the threshold value and then confirm whether this determination method is medically valid.

\section{Acknowledgments}

This work was supported in part by Artificial Intelligence Research Promotion Foundation.

\section{References}

[1] M Kachuee et al., "ECG Heartbeat Classification: A Deep Transferable Representation", 2018 IEEE International Conference on Healthcare Informatics, Jul. 2018

[2] S.Kiranyaz et al., "Convolutional Neural Networks for Patient Specific ECG Classification", 37th Annual International Conference of the IEEE Engineering in Medicine and Biology Society, pp.2608-3611. Aug. 2015

[3] S.van den Elzen et al., "Reducing snapshots to points: A visual analytics approach to dynamic network exploration", IEEE Transactions on Visualization and Computer Graphics, Vol. 22, No. 1, pp.1-10 Aug. 2015

[4] MIT-BIH Arrhythmia Database. https://physionetorg/content/mitdb/1.0.0/

Address for correspondence:

Shota Hori.

Graduate School of Engineering, Oita University, 700 Dannoharu, Oita 870-1192, Japan

v19e3019@oita-u.ac.jp 